\title{
Smart Grid Technologies as a Concept of Innovative Energy Development: Initial Proposals for the Development of Ukraine
}

\author{
Vladyslav Pliuhin ${ }^{1}$ (D), Vitaliy Teterev ${ }^{1}$ (D), and Anatolii Lapko ${ }^{2}$ (D) \\ 1 O. M. Beketov National University of Urban Economy in Kharkiv, Kharkiv, Ukraine \\ 2 JSC National Power Company Ukrenergo, Kyiv, Ukraine
}

\author{
Article History \\ Received: \\ 06 August 2021 \\ Accepted: \\ 29 September 2021 \\ Published online: \\ 29 October 2021 \\ Keywords
Smart Grid;
Intelligent Measure-
ment;
SCADA;
Data Science;
Big Data;
Machine Learning
}

\begin{abstract}
The formation of the concept of Smart Grid is associated with a number of issues, including theoretical and methodological. One of the main problems in forming such theory is to build its basis, the starting point for the development of which is the definition of Smart Grid as a systems of views concept on the future of power engineering, the principles of operation and technological basis of which undergoes significant changes compared to modern energy. The paper is aimed at reviewing and developing directions and approaches to the definition of Smart Grid in combination with machine learning mechanisms, highlighting their diverse and common nature to develop a holistic innovative energy development. In this paper, a study of the energy sector in Ukraine was conducted. Its efficiency and innovative development are considered. Problems with the implementation of Smart Grid technology, which arise when using alternative sources and monitoring and administration systems, were highlighted. The ways of mathematical formulation of the Smart Grid optimization problem are determined using the Data Science approach based on the machine learning system and neural networks. Big Data processing methods, Data Mining, statistical methods, artificial intelligence methods, and Machine Learning are analyzed. The design and development of databases and application software will be done using the Data Science method. Smart-technologies will take over the processes of control, accounting and diagnostics of assets, which will provide promising opportunities for self-recovery of the power system, as well as efficient operation of fixed assets. With the introduction of Smart Grid technologies for the Ukrainian power industry, significant fundamental changes will take place. This is the transition from centralized methods of generation and transmission of electricity to distributed networks with the ability to control energy production facilities and network topology at any point, including at the consumer level. Replacement of centralized demand forecasting according to the methodology of active consumer influence becomes an element and subject of the management system. A high-performance information and computing infrastructure will be built as the core of the energy system. This approach creates the preconditions for the widespread introduction of new devices that increase the maneuverability and controllability of the equipment. The creation of next-generation operational applications (SCADA/EMS/NMS) allows the use of innovative algorithms and methods of power system management, including its new active power elements.
\end{abstract}

\section{INTRODUCTION}

Currently, there is a growing interest in the rapidly developing in the last decade in the world of electricity conversion Smart Grid ("smart grid", "smart power system"). Smart Grid in the world is a common term, an unambiguous interpretation of which, however, has not been developed [1].
Many foreign countries reflect different views and positions on Smart Grid, which is primarily due to differences in goals and expectations from the development of this area among a wide range of stakeholders:

1) at the state level in most countries Smart Grid is seen as the ideological basis of national programs for the development of electricity, which provide for its transformation in the di-

Corresponding author: vitaliy.teterev@kname.edu.ua (Vitaliy Teterev)

(C) 2021 The Author(s). Published by O. M. Beketov National University of Urban Economy in Kharkiv Use permitted under Creative Commons Attribution 4.0 International (CC BY 4.0)

Cite as: Pliuhin, V., Teterev, V., \& Lapko, A. (2021). Smart Grid technologies as a concept of innovative energy development: initial proposals for the development of Ukraine. Lighting Engineering \& Power Engineering, 60(2), 47-65. https:// doi.org/10.33042/2079-424X.2021.60.2.02 
rection of forming a new customer-oriented model of operation;

2) companies-manufacturers of equipment and technology evaluate this area in terms of opening opportunities for the creation and development of new business, the formation of a market niche for their companies;

3) companies view Smart Grid as a base to ensure their sustainable development based on innovation.

The results of the study allowed to formulate an approach in which Smart Grid is proposed to be understood as a concept and system of views on the future of electricity, the principles of operation and technological basis of which undergo significant changes compared to the characteristics of modern energy.

The paper pays attention to ideological and conceptual-methodological issues, the solution of which will contribute to the formation of the foundations for further development of energy in Ukraine on the basis of Smart Grid.

The most important aspects of the implementation of this concept are the creation and development of a new technological base. That will allow to form its basis of concrete technologies, methods, tools, for cooperation of the scientific and industrial and technological institutes which are carrying out new developments, with the electric power companies, implement and use such developments in manufacture.

In recent years, the implementation of programs and projects in the direction of Smart Grid, which cover a wide range of problems and tasks, embarked on the vast majority of industrialized countries, as well as many developing countries.

The scale, direction, intensity and pace of this activity in different countries are different, they are largely determined by the degree of diversity of elements of the energy system, the development of functions such as interaction with consumers, typical methods of combining small energy sources, including non-traditional, other factors.

One of the main initiators and investors of Smart Grid in most countries is the state. Significant interest in participating in programs and projects deployed in this direction is shown by large companies - manufacturers of electrical equipment, as well as companies working in the field of information and communication technologies, which carry out research and development in various fields related to Smart Grid [2].

Smart Grid and Smart Metering are presented as a priority in the development of energy in the near future in the EU, USA, China and other countries [3].

In first place in terms of investment in Smart Grid (interval - 5 years) - China (\$ 9 billion), in sec- ond place - the United States (\$ 7 billion), in third place - Japan (\$ 900 million) [4].

Today, the most advanced in this area is the United States. In parallel, they work on the development of technologies, standards, evaluation of the effectiveness of the implementation of intelligent energy networks, as well as explore how this will further affect the economy and security of the country.

The introduction of smart grid systems will contribute to more efficient use of energy, increase the share of renewable energy sources in the energy balance, as well as reduce energy infrastructure through distributed generation and reduce overall energy consumption. [5]

Extensive decentralization of generation costs the development of distributed generation (primarily due to renewable energy sources) and the tendency to reduce the number of powerful generation facilities (such as nuclear power plants and thermal power plants) requires completely new approaches to managing complex energy networks, as well as new requirements to increase the manageability and reliability of energy networks.

Thus, today in the United States there are 12 million small distributed generation units with a total installed capacity of $220 \mathrm{GW}$ (at a growth rate of $5 \mathrm{GW}$ per year). In the EU, distributed generation averages about $10 \%$ of total electricity production (45\% in Denmark) [6].

Smart Grid - is a tool for managing distributed generation and organizing intelligent interaction of generation with consumer objects.

In addition, Smart Grid expands the capabilities of consumers, making them more aware of energy use, and also allows you to respond to price signals from the energy market.

\section{DEVELOPMENT OF SMART GRID AND SMART METERING IN EU MEMBER STATES}

In order to promote the development of Smart Grid, the European Commission encourages the deployment of Smart Grid and its main component - Smart Metering in all EU Member States, in line with the 2009 recommendations in the field of electricity and gas [1].

In 2009, the European Commission set up a Smart Grids task force to develop common standards and specifications for smart grids.

The 2009 Directive recommended the widespread introduction of Smart Metering in all EU countries.

The European Commission has defined a minimum set of ten functional requirements to ensure interoperability and the possibility of technological updates Smart Metering [7]. 
Smart Metering - in the power industry. According to reports submitted and processed by the European Commission, the CBA analysis on Smart Metering for electricity in most EU member states has yielded positive results. Then began an active process of large-scale implementation of national systems in this direction, which by 2022 should cover $80 \%$ of all electricity metering equipment $[8,9]$.

In Germany, it is planned to use Smart Metering selectively only for consumers with a high level of energy use or to install only on newly introduced facilities.

In Ukraine at the moment electrical networks operate on the principle (generator - systemforming electrical networks - distribution electrical networks - consumers) [10]. System-forming networks in most cases are looped, and electrical distribution networks consist of radial lines with oneway power supply. The concept of "intelligent" electrical network offers a different principle of construction [11, 12]. It is a system of generator transmission line - consumer, but the consumer is involved in the production and redistribution of energy. The development of RES stimulates a green tariff.

Recent events in the country have led to shortages and significant increases in fuel costs, which stimulates the development of alternative sources of electricity. That is, generating capacity in the future power system will be more distributed than concentrated, as now.

The peculiarity of alternative sources of electricity is their relatively small (current 200-250 MW, projects up to $700 \mathrm{MW}$ ) capacity and the instability of the power parameters generated. Obviously, to stabilize the parameters of such sources and their automatic synchronization with the network requires a very "smart" control devices. Coal and gas reserves are insufficient, and the development of renewable energy sources with their subsequent inclusion in the country's unified energy system is a strategically important task.

In Ukraine as in the rest of the world, the problem of energy depletion, such as coal, natural gas, peat, and uranium ore, is very acute. According to statistics, fossil fuel reserves will be enough for humanity for about $60-80$ years [13]. Today, there are several strategic directions in the world regarding the prospects of heat and electricity. It is necessary to allocate the most important of them:

1) development of new methods of obtaining electricity (for example, the project to create a thermonuclear reactor ITER);

2) improving the technological cycle of existing thermal, nuclear and hydraulic power plants through the modernization of basic and auxiliary equipment, equipment of the system of own needs, the introduction of the latest digital automated process control systems (ACS $\mathrm{TP})$;

3) increasing the share of renewable energy sources (RES) and energy storage devices (NEs) in electricity systems (UES);

4) introduction of the concept of "smart" or "intelligent" power system (Smart Grid) in the principles of management of energy systems with a larger share of RES;

5) increasing the stability of the power system through the use of relay protection and emergency automation (RZA), built using the latest hardware and protective logic;

6) development and implementation of various ways to save or save electricity (various energy-saving technologies, Smart House, Smart Metering, etc.) [14].

Following the accident at the Fukushima nuclear power plant (NPP) in Japan in the spring of 2011 [15], the continued use of nuclear energy in many countries around the world is in question. For example, in Germany, within ten years, all existing nuclear power plants must be decommissioned.

In Ukraine, such solutions are not acceptable, as nuclear power plants produce more than $50 \%$ of all energy, and are strategic structural elements of the energy system. An alternative way out of this situation is the large-scale introduction of RES. An example of such an approach is Germany, according to an analysis by the Fraunhofer Society for Solar Energy Systems, the share of renewable energy sources (RES) in the structure of electricity production in Germany in 2019 was $46 \%$, and on some days it exceeded $65 \%[16,17]$. Reducing the use of coal has helped protect the climate. Compared to the previous year, the share of electricity generated by burning brown coal decreased by $22 \%$, and coal - by $33 \%$. However, it should be noted that the generated power by wind power plants (WPPs), solar power plants (WPPs), other alternative energy sources is not a constant value and depends on a number of natural conditions: the presence of wind, solar radiation activity and more. In this case, such instability of RES generation makes its negative adjustments to the sustainable operation of the power system. The classical principle of organization of power systems management is not suitable for power plants with a large share of RES.

Smart Grid is the use in the process of organizing and managing the latest technologies and algorithms, such as virtual power plants (WPP), FACTS systems, phasor or PMU (Phasor Measurement Unit), DC inserts (HDVC), various types of energy storage (including numbers and electric cars), etc. [18].

The construction of communication systems, telecommunications, control systems and relay protec- 
tion systems is based on the use of the IEC (IEC) 61850 protocol.

Nuclear power plants also serve as bases for Ukraine. It should be noted the trend of modernization of domestic TPPs and NPPs (DTEK, NJSC Energoatom, etc.), built in Soviet times, as well as the trend of design and future construction of new generation units of Ukrainian TPPs with a nominal capacity of $320 \mathrm{MW}$ to $660 \mathrm{MW}$.

In Ukraine, work on the introduction of new technologies in the national energy system has begun relatively recently. For example, since 2014, the Belgian company Tractebel has been developing and implementing a number of pilot technologies and Smart Grid projects at the level of the system operator - NEC Ukrenergo. Distribution system operators are also gradually trying to implement elements of smart electrical networks.

DTEK is the most active in implementing technologies. The company said it is actively installing smart meters and automating the power system with state-of-the-art software. This will help her to "see" accidents faster and, as a result, eliminate them much faster.

The share of renewable electricity in Ukraine as of June 1, 2021 is about $13.1 \%$ of the total installed generation capacity. This fact is explained only by the fact that domestic alternative energy is at an early stage of development.

It is also possible to emphasize the high cost of RES and their maintenance. However, despite this, such large companies as DTEK, Active-Solar, and Fuhrlnder-Wind Technologies are pursuing an active policy of introducing and promoting renewable energy sources in Ukraine.

Today there are two main scenarios for the development of energy systems [19]:

- increase of reliability at the expense of reserve connection of other type of sources for minimization of damage at emergency events. In this case, the bandwidth, resource of the main elements in nominal modes will be used minimally, which will lead to a relative increase in the cost of network infrastructure;

- "intellectualization" of the electrical network, associated with a combination of comprehensive tools for management, control, monitoring and communication allows to ensure much higher productivity and reliability of the network, improving energy quality. When implementing Smart Grid technologies in Ukraine, it is important to consider possible scenarios.

Thus, when using alternative sources as a conventional power plant, there are many technical issues regarding the integration of a non-permanent source into the power system with fixed parameters.

\section{PROBLEMS IMPLEMENTING SMART GRID}

Interruptions in generation at work put forward special requirements to work of Smart Grid technologies. With the use of alternative sources centrally on a global scale, the problems increase. With an isolated approach - localized and become much easier. The introduction of Smart Grid technology also means a fundamental reorganization of the power industry [20]:

- ensuring uninterrupted operation of the electrical network in conditions of increasing load;

- reduction of electricity losses due to the construction of "intelligent" metering systems with the possibility of accounting for electricity quality and load limitation;

- development of a communication environment capable of reliably and qualitatively supporting bidirectional information exchange between suppliers and consumers of energy resources [21].

\section{FORMATION OF THE RESEARCH TASK: WHAT IS PLANNED TO BE DONE}

One way to solve this task is the use of wireless intelligent communication devices:

- improving the quality of electricity through the use of reactive power compensation devices;

- the use of "intelligent" equipment and software for network topology management in order to ensure the reliability of operation;

- the use of large-capacity energy storage devices to balance the load schedule, as well as to ensure the smooth operation of particularly important facilities;

- development of market relations in the energy business with the involvement of electricity consumers (creation of separate sections of the network analogous to micronetworks) as possible suppliers of electricity at the right time in the right part of the network;

- development and production by domestic companies of high-tech competitive products to ensure the functioning of the "intelligent" electrical network;

- development of distributed energy, including cogeneration through the modernization of existing boilers, to cover maximum loads and eliminate energy shortages. Features of innovative transformation of electric power industry with introduction of Smart Grid technologies are based on the following initial positions; 
- system modernization of the industry covers all its components: electricity generation, transmission and distribution (including in the utilities sector), sales and dispatching;

- the energy system is developing as an Internet-like infrastructure to support energy, information, economic and financial relationships between energy market players and other stakeholders;

- electrical network (all its segments) are considered as the main object of formation of a new technological base, which allows to significantly improve the old and create new functional properties of the power system, ensuring the greatest achievement of key objectives identified by joint choice of all stakeholders [14].

\section{ANALYSIS OF THE CURRENT SITUATION OF ENERGY PRODUCTION IN UKRAINE}

We can analyze the consumption and production of electricity in recent years based on the data given in the graphs and tables from 2019 to 2020 [22].

At the end of 2020, the UES of Ukraine combined thermal, nuclear, hydraulic, wind and solar power plants, as well as biofuel stations with a total installed capacity of $54,771.2 \mathrm{MW}$ is given in Table 1 . Information on the installed capacity and number of power units of nuclear, thermal and hydraulic power plants is provided in Tables 2-4.

The structure of the installed capacity of power plants as of the end of 2020 by regions of the UES of Ukraine is given in Table 5. The list of wind farms and SES in UES of Ukraine with installed capacity over $50 \mathrm{MW}$ is given in Tables 6-7.

The monthly installed capacity of renewable energy sources of SES and UES of Ukraine for 20192020 is provided in Fig. 1 and Fig. 2.

As of the end of the year, there are 29,931 SES of households in Ukraine with a total installed capacity of $778.7 \mathrm{MW}$, which is $14.5 \%$ of the total installed capacity of SES in the country's energy system (data from the official website of the State Agency for Energy Efficiency and Energy Saving of Ukraine).

In 2020, the installed capacity of wind farms and SES is $1.11 \mathrm{GW}$ and $5.98 \mathrm{GW}$, and the actual production is 2.57 billion $\mathrm{kWh}$ and 5.78 billion $\mathrm{kWh}$, respectively. The largest in wind generation are DTEK VDE (45\%); Windcraft Ukraine LLC (30\%); LLC "Wind Parks of Ukraine" (14\%); NBT AS (4\%). The leaders in the SES segment are DTEK VDE (10\%); VR Capital (8.9\%); CNBM (5\%); UDP Group (2.2\%); Scatec Solar $(2 \%)$; TIU Canada $(1 \%)$.

In total, the alternative energy sector of Ukraine has 881 business entities for which "green tariffs" have been set for electricity generated at electricity facilities.

Table 1. The capacity of UES power plants in Ukraine has been installed as of 31.12.20

\begin{tabular}{lcccc}
\hline & \multicolumn{2}{c}{$12 / 31 / 2019$} & \multicolumn{2}{c}{$12 / 31 / 2020$} \\
\cline { 2 - 5 } & Power, MW & $\begin{array}{c}\text { Share of total } \\
\text { installed capacity, } \%\end{array}$ & Power, MW & $\begin{array}{c}\text { Share of total } \\
\text { installed capacity, } \%\end{array}$ \\
\hline TES GK & 21842.0 & 41.37 & 21842.0 & 39.88 \\
CHP and cogeneration units & 4335.5 & 8.21 & 4344.8 & 7.93 \\
SRF manufacturers * & 1755.6 & 3.33 & 1759.7 & 3.21 \\
NPP & 13835 & 26.21 & 13835 & 25.26 \\
HPP & 4809.1 & 9.11 & 4828.7 & 8.82 \\
PSP & 1487.8 & 2.82 & 1487.8 & 2.72 \\
SES (including households) & 3555.4 & 6.74 & 5362.6 & 9.79 \\
WEIGHT & 1025.0 & 1.94 & 111.2 & 2.03 \\
Biofuel stations & 142.5 & 0.27 & $\mathbf{1 9 9 . 5}$ & 0.36 \\
\hline \multicolumn{1}{c}{ Total } & $\mathbf{5 2 7 8 7 . 9}$ & $\mathbf{1 0 0 . 0 0}$ & $\mathbf{5 4 7 7 1 . 0}$ & $\mathbf{1 0 0 . 0 0}$ \\
\hline
\end{tabular}

* - manufacturers located on the territory of the licensed activity of the distribution system operator.

Table 2. Nuclear power plants of Ukraine

\begin{tabular}{lccc}
\hline & $\begin{array}{c}\text { Installed capacity } \\
\text { at the end of 2020 p., MW }\end{array}$ & $\begin{array}{c}\text { Number and capacity of } \\
\text { power units, pcs/MW }\end{array}$ & Reactor type \\
\hline Zaporizhzhya NPP & 6000 & $6 \times 1000$ & WWER-1000/320 \\
& & & WWER-1000/302 \\
South Ukrainian NPP & 3000 & $3 \times 1000$ & WWER-1000/338 \\
& & & WWER-1000/320 \\
Rivne NPP & & $2 \times 1000$ & WWER-1000/320 \\
& 2835 & $1 \times 420$ & WWER-440/213 \\
Khmelnitsky NPP & & $1 \times 415$ & WWER-440/213 \\
\hline Total & 2000 & $2 \times 1000$ & WWER-1000/320 \\
\hline
\end{tabular}


Table 3. The largest thermal power plants and thermal power plants in Ukraine

\begin{tabular}{|c|c|c|}
\hline & Total installed capacity, MW & $\begin{array}{c}\text { Number and installed capacity } \\
\text { of power units pcs/MW }\end{array}$ \\
\hline JSC DTEK DHPROEHERGO & 5839 & $18 / 5839$ \\
\hline Zaporizhzhya TPP & 2850 & $\begin{array}{l}2 \times 800 ; 2 \times 325 \\
2 \times 300\end{array}$ \\
\hline Kryvyi Rih TPP & 2079 & $\begin{array}{c}2 \times 282 ; 4 \times 300 \\
1 \times 315\end{array}$ \\
\hline Prydniprovska TPP & 910 & $1 \times 310 ; 4 \times 150$ \\
\hline PJSC “DONBASENERGO" & 880 & $1 / 800$ \\
\hline Slavic TPP & 880 & $\begin{array}{c}1 \times 800 \\
(1 \text { turn }-80 \mathrm{MW})\end{array}$ \\
\hline PJSC “CETREHERGO” & 7695 & $23 / 7695$ \\
\hline Coal TPP & 3600 & $\begin{array}{l}4 \times 300 ; 3 \times 800 \\
2 \times 175 ; 2 \times 180\end{array}$ \\
\hline Zmiiv TPP & 2270 & $\begin{array}{c}1 \times 185 ; 1 \times 190 ; \\
1 \times 280 ; 2 \times 290 \\
1 \times 325\end{array}$ \\
\hline Trypillya TPP & 1825 & $5 \times 300 ; 1 \times 325$ \\
\hline JSC DTEK ZAHIDEHERGO & 4676 & $20 / 4476$ \\
\hline Burshtyn TPP & 2366 & $\begin{array}{c}1 \times 215 ; 1 \times 210 \\
1 \times 206 \\
7 \times 195 ; 2 \times 185\end{array}$ \\
\hline Dobrotvirska TPP & 510 & $\begin{array}{c}1 \times 160 ; 1 \times 150 \\
(1 \text { turn }-2 \times 100 \mathrm{MW})\end{array}$ \\
\hline Ladyzhynska TPP & 1800 & $6 \times 300$ \\
\hline DTEK SKHIDENERGO LLC & 2752 & $13 / 2752$ \\
\hline Kurakhivska TPP & 1532 & $\begin{array}{l}4 \times 225 ; 1 \times 222 ; \\
1 \times 210 ; 1 \times 200\end{array}$ \\
\hline Luhansk TPP & 1220 & $2 \times 210 ; 4 \times 200$ \\
\hline TES GK & 21842 & $75 / 21562$ \\
\hline KP “KYIVTEPLOENERGO” & 1200 & $6 / 1200$ \\
\hline Kyiv CHP-5 & 700 & $2 \times 250 ; 2 \times 100$ \\
\hline Kyiv CHP-6 & 500 & $2 \times 250$ \\
\hline KHARKIV CHP-5 & 470 & $\begin{array}{l}1 \times 250 \\
2 \times 110\end{array}$ \\
\hline Total & 23512 & $84 / 23232$ \\
\hline
\end{tabular}

Table 4. The largest hydraulic power plants in Ukraine

\begin{tabular}{|c|c|c|}
\hline & $\begin{array}{l}\text { Installed capacity at } \\
\text { the end of } 2020 \text { p., MW }\end{array}$ & $\begin{array}{c}\text { Number and capacity } \\
\text { of hydraulic turbines, pcs/MW }\end{array}$ \\
\hline Kyiv HPP & 440.0 & $20 \times 22.0$ \\
\hline Kaniv HPP & 500.0 & $\begin{array}{c}8 \times 18.5 \\
16 \times 22.0\end{array}$ \\
\hline Kremenchug HPP & 700.4 & $\begin{array}{l}7 \times 57.2 \\
5 \times 60.0\end{array}$ \\
\hline Middle Dnieper HPP & 388.0 & $\begin{array}{l}6 \times 50.0 \\
2 \times 44.0\end{array}$ \\
\hline $\begin{array}{l}\text { Kakhovka HPP } \\
\text { Dniester HPP }\end{array}$ & $\begin{array}{l}343.2 \\
702.0\end{array}$ & $\begin{array}{c}6 \times 57.2 \\
6 \times 117.0\end{array}$ \\
\hline Dnieper HPP-1 & 650.6 & $\begin{array}{c}9 \times 72.0 \\
1 \times 2.6 \\
3 \times 104.5\end{array}$ \\
\hline Dnieper HPP-2 & 912.5 & $\begin{array}{l}4 \times 120.0 \\
1 \times 119.0\end{array}$ \\
\hline Total HPP & 4636.7 & $94 / 4636.7$ \\
\hline Kyiv PSP & 213.8 & $\begin{array}{l}5 \times 33.4 \\
1 \times 46.8\end{array}$ \\
\hline Dniester PSP & 972.0 & $3 \times 324.0$ \\
\hline Tashlyk PSP & 302.0 & $2 \times 151.0$ \\
\hline Total PSP & 1487.8 & $11 / 1487.8$ \\
\hline
\end{tabular}


Table 5. The structure of the installed capacity by regions of the UES of Ukraine at the end of 2020

\begin{tabular}{|c|c|c|c|c|c|c|c|c|c|c|c|c|c|c|c|}
\hline \multirow[t]{2}{*}{ 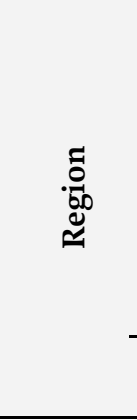 } & \multicolumn{3}{|c|}{ НРP } & \multicolumn{2}{|c|}{ PSP } & \multicolumn{2}{|c|}{ TES GK } & \multicolumn{2}{|c|}{$\begin{array}{c}\mathrm{CHP} \text { and } \\
\text { cogeneration } \\
\text { units }\end{array}$} & \multicolumn{2}{|c|}{ NPP } & \multicolumn{2}{|c|}{ 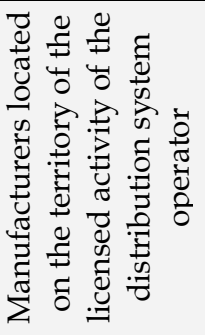 } & \multicolumn{2}{|c|}{$\begin{array}{c}\text { SES, WPP } \\
\text { and } \\
\text { biomass }\end{array}$} \\
\hline & $\begin{array}{l}P_{\text {inst }} \\
\text { MW } \\
\end{array}$ & $\begin{array}{l}\text { HPP, } \\
\text { MW }\end{array}$ & $\begin{array}{l}\% \text { of } \\
P_{\text {inst }} \\
\end{array}$ & $\begin{array}{l}\text { PSP, } \\
\text { MW } \\
\end{array}$ & $\begin{array}{l}\% \text { of } \\
P_{\text {inst }} \\
\end{array}$ & $\begin{array}{l}P_{\text {inst }} \\
\text { MW } \\
\end{array}$ & $\begin{array}{l}\% \text { of } \\
P_{\text {inst }} \\
\end{array}$ & $\begin{array}{l}P_{\text {inst }} \\
\mathrm{MW}\end{array}$ & $\begin{array}{l}\% \text { of } \\
P_{\text {inst }} \\
\end{array}$ & $\begin{array}{l}P_{\text {inst }} \\
\text { MW }\end{array}$ & $\begin{array}{l}\% \text { of } \\
P_{\text {inst }} \\
\end{array}$ & $\begin{array}{l}P_{\text {inst, }} \\
\text { MW }\end{array}$ & $\begin{array}{l}\% \text { of } \\
P_{\text {inst }} \\
\end{array}$ & $\begin{array}{l}P_{\text {inst }} \\
\mathrm{MW} \\
\end{array}$ & $\begin{array}{l}\% \text { of } \\
P_{\text {inst }} \\
\end{array}$ \\
\hline $\begin{array}{l}\text { South - } \\
\text { West }\end{array}$ & 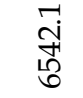 & 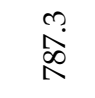 & $\stackrel{\circ}{\stackrel{ }{7}}$ & Nૂ & $\begin{array}{l}\infty \\
\infty \\
+ \\
+\end{array}$ & $\stackrel{8}{\infty}$ & ลุ & & & ஓ্ণ & $\begin{array}{l}\text { مि } \\
\text { कें }\end{array}$ & ọ & $\stackrel{0}{0}$ & ָ̃ & $\stackrel{+}{+}$ \\
\hline $\begin{array}{c}\text { Dniprov } \\
\text { sky }\end{array}$ & 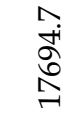 & $\underset{\infty}{\infty}$ & $\stackrel{\circ}{\circ}$ & & & $\begin{array}{l}\text { के } \\
\infty \\
\text { மn }\end{array}$ & $\begin{array}{l}8 \\
\ddot{\infty}\end{array}$ & $\stackrel{0}{\infty}$ & 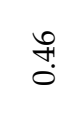 & 8 & $\begin{array}{l}\sigma \\
\dot{m}\end{array}$ & $\widehat{N}$ & $\underset{+}{+}$ & $\begin{array}{l}\text { ọं } \\
\text { ঠे }\end{array}$ & 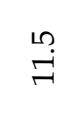 \\
\hline Central & 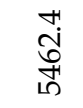 & $\begin{array}{l}\text { ஸุ' } \\
\text { ڤn }\end{array}$ & $\stackrel{\text { ำ }}{\stackrel{\overbrace{}}{\rightleftharpoons}}$ & $\begin{array}{l}\infty \\
\stackrel{\infty}{N}\end{array}$ & के & $\stackrel{\stackrel{1}{2}}{\stackrel{\infty}{\sim}}$ & $\begin{array}{l}\text { F! } \\
\text { ले }\end{array}$ & 홍 & $\begin{array}{l}\infty \\
\text { ம் }\end{array}$ & & & $\stackrel{0}{\stackrel{0}{\rightleftharpoons}}$ & సี & $\begin{array}{l}+1 \\
\stackrel{H}{10}\end{array}$ & $\stackrel{\text { L }}{\stackrel{2}{\circ}}$ \\
\hline West & $\begin{array}{l}\text { ․ } \\
\text { م્ } \\
\stackrel{N}{N}\end{array}$ & 아 & ڤై & & & $\begin{array}{l}\stackrel{0}{N} \\
\stackrel{\infty}{N}\end{array}$ & ने & ભొ & $\begin{array}{l}\stackrel{20}{\digamma} \\
\stackrel{+}{+}\end{array}$ & $\begin{array}{l}L_{\infty} \\
\text { d } \\
\text { N }\end{array}$ & $\begin{array}{l}\sigma \\
\infty \\
\infty \\
\infty\end{array}$ & 吕 & ๙ & न̆ं & 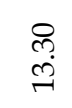 \\
\hline Southern & $\begin{array}{l}\text { 로 } \\
\stackrel{2}{10}\end{array}$ & $\stackrel{+}{+}$ & 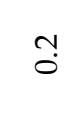 & ठ઼ & $\begin{array}{l}\text { กิ } \\
\text { เก }\end{array}$ & & & ஜ̊ํ & 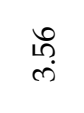 & ஓి & $\begin{array}{l}\infty \\
\dot{\infty} \\
\dot{\Gamma}\end{array}$ & $\begin{array}{l}\text { Nִ } \\
\stackrel{1}{ }\end{array}$ & $\stackrel{\stackrel{2}{+}}{+}$ & ત્்̀ & $\begin{array}{l}\alpha \\
\text { ஸे }\end{array}$ \\
\hline $\begin{array}{l}\text { North- } \\
\text { ern }\end{array}$ & 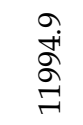 & & 룽 & & & $\begin{array}{l}\text { నิ } \\
\text { ถู }\end{array}$ & స̃ & $\underset{\infty}{\vec{D}}$ & 올 & & & $\begin{array}{l}\text { 혀 } \\
\text { N̦ }\end{array}$ & $\stackrel{+}{+}$ & $\begin{array}{l}\text { 형 } \\
\text { \& }\end{array}$ & ִָ \\
\hline $\begin{array}{l}\text { UES of } \\
\text { Ukraine }\end{array}$ & ז̃ & 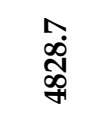 & $\begin{array}{l}N \\
\infty \\
\infty\end{array}$ & $\begin{array}{l}\text { 我 } \\
\stackrel{\infty}{\infty} \\
\stackrel{+}{+}\end{array}$ & $\stackrel{N}{N}$ & $\begin{array}{l}\stackrel{\circ}{\mathcal{N}} \\
\stackrel{+}{\infty} \\
\stackrel{\text { N }}{ }\end{array}$ & $\begin{array}{l}\infty \\
\infty \\
\text { ஸ் }\end{array}$ & $\begin{array}{l}\infty \\
\stackrel{+}{+} \\
\mathscr{H}\end{array}$ & જ̆ & 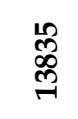 & $\begin{array}{l}\text { ণุ } \\
\text { ஸे }\end{array}$ & $\begin{array}{l}\hat{\imath} \\
\text { مิ } \\
\text { ลิ }\end{array}$ & $\begin{array}{r}\overline{ } \\
\text { ஸे }\end{array}$ & సֶ. & $\stackrel{\infty}{\stackrel{\infty}{\sim}}$ \\
\hline
\end{tabular}

Table 6. List of wind farms in the UES of Ukraine with an installed capacity of over $50 \mathrm{MW}$

\begin{tabular}{llcc}
\hline No. & \multicolumn{1}{c}{ Power plant } & Region & Installed capacity, MW \\
\hline 1 & Botievskaya WPP (WindPower LLC) & Dniprovsky & 199.87 \\
2 & LLC “Windcraft Kalanchak“ Myrnenskaya wind farm & Southern & 163 \\
3 & Primorskaya Wind Power Plant LLC & Dniprovsky & 99.58 \\
4 & Primorskaya Wind Power Plant-2 LLC & Dniprovsky & 99.58 \\
5 & LLC "Orlivska Wind Power Plant" & Dniprovsky & 98.8 \\
6 & Novotroitskaya WPP (1-5, 9th turn) Windcraft Tavria LLC & Southern & 69.3 \\
7 & Windcraft Ukraine LLC Overyanivska WPP & Southern & 68.4 \\
8 & Ochakivsky Wind Park LLC & Southern & 57.1 \\
\hline & Total WPP/WPP over 50 MW & $\mathbf{1 1 1 1 . 2 / 8 5 5 . 6 3}$ \\
\hline
\end{tabular}

Table 7. List of SES in the UES of Ukraine with an installed capacity of over $50 \mathrm{MW}$.

\begin{tabular}{|c|c|c|c|}
\hline No. & Power plant & Region & Installed capacity, MW \\
\hline 1 & SES Pokrovska (LLC “Solar Pharm-3”) & Dniprovsky & 240 \\
\hline 2 & LLC “Solar Pharm 1“ (Nikopol SES) & Dniprovsky & 200 \\
\hline 3 & SES “Morozivka" (LLC “Sanvin 5") & Dniprovsky & 70 \\
\hline 4 & SES “Energopark Yavoriv" (1, 2 turns) & West & 63.36 \\
\hline 5 & LLC “Green Energy Tokmak" 1-9 p.k. & Dniprovsky & 56.97 \\
\hline 6 & FES "Kamyanets-Podilska" & Southwestern & 50.0 \\
\hline & $\begin{array}{c}\text { Total SES / SES over } 50 \mathrm{MW} \\
\% \text { of the total installed capacity of SES }\end{array}$ & & $\begin{array}{c}5362.6 / 680.33 \\
12.7 \%\end{array}$ \\
\hline
\end{tabular}




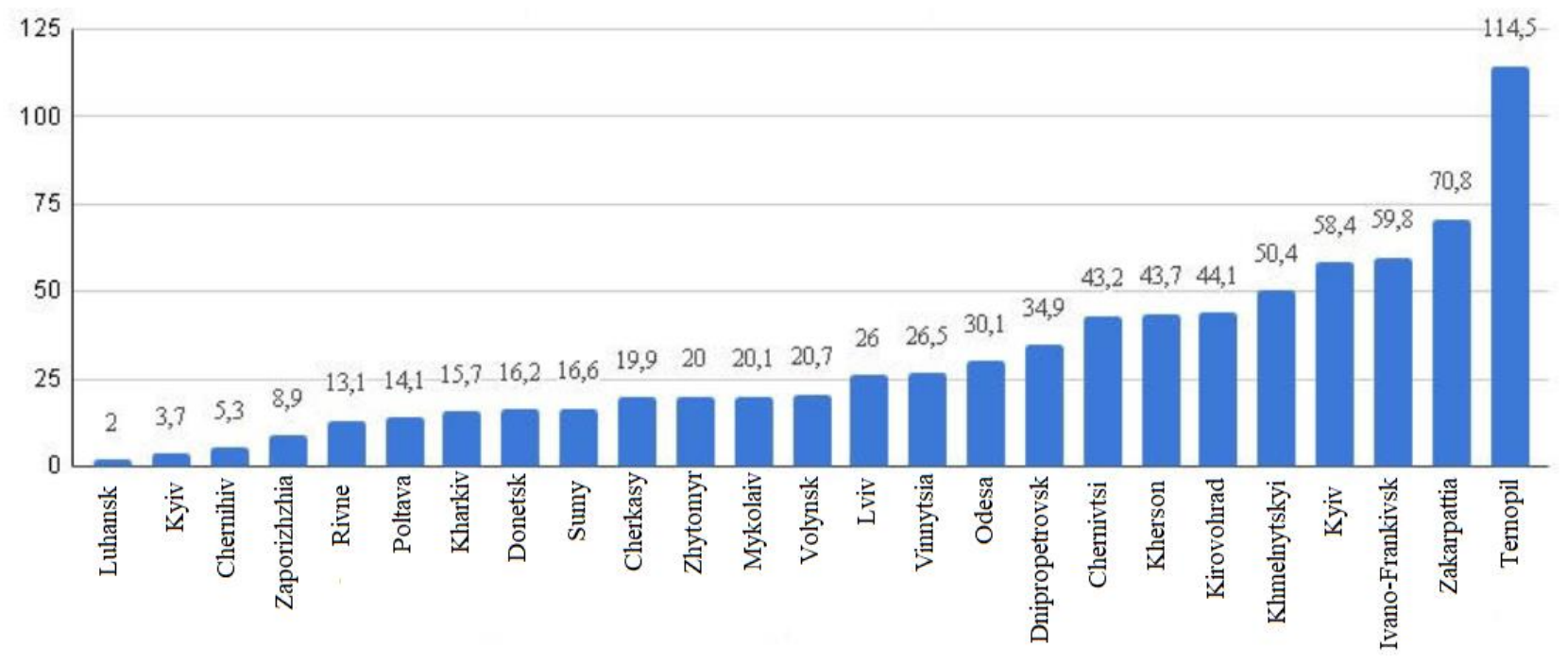

Figure 1. Installed capacity of SES households at the end of 2020, MW

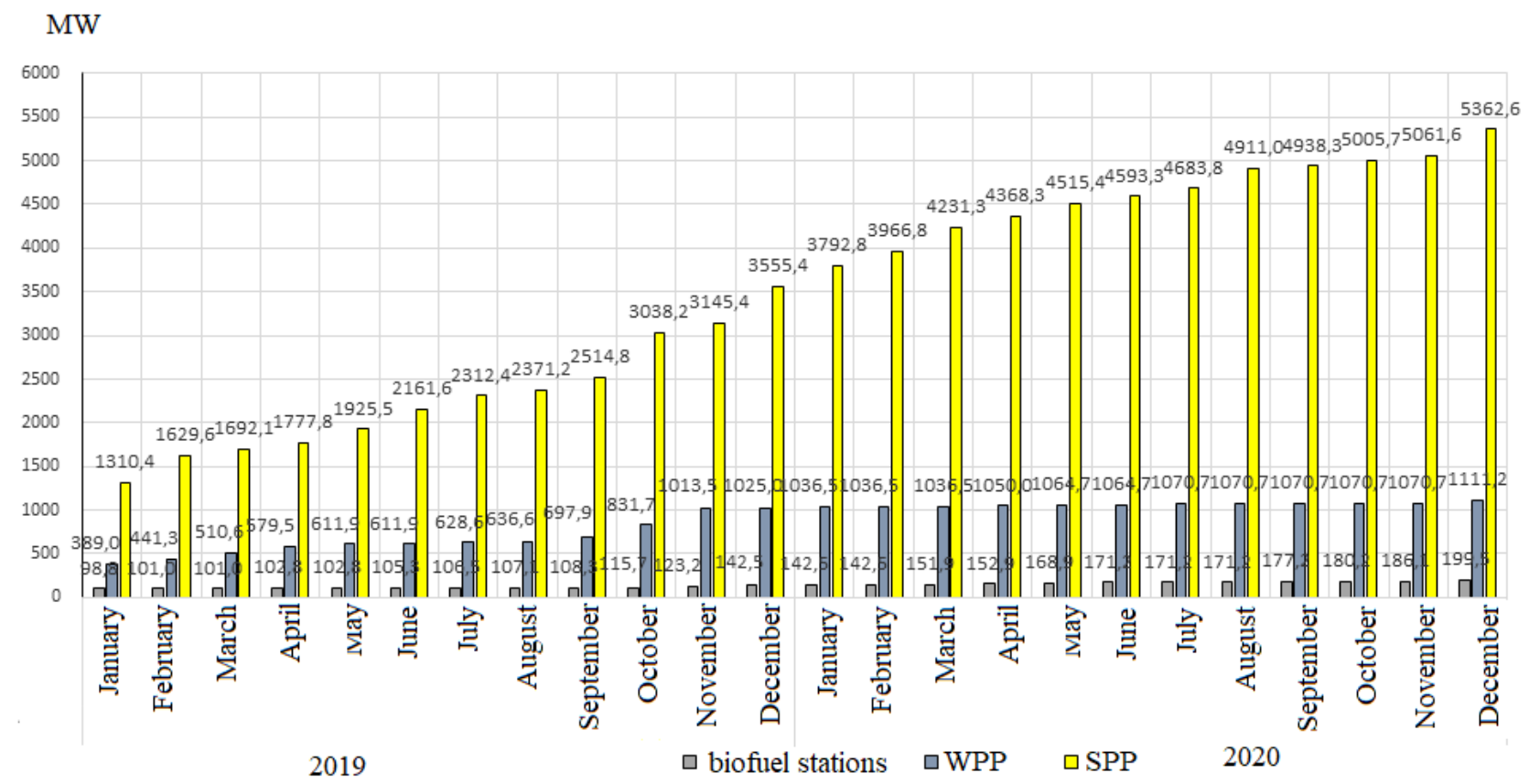

Figure 2. Monthly installed capacity of renewable UES of Ukraine energy sources for 2019-2020

Table 8. Structure of electricity production in the first half of 2021

\begin{tabular}{|c|c|c|c|c|c|c|}
\hline \multirow{2}{*}{ Electricity generation } & \multicolumn{2}{|c|}{2020} & \multicolumn{2}{|l|}{2021} & \multicolumn{2}{|c|}{ Relative to 2020} \\
\hline & million $\mathrm{kWh}$ & $\%$ & million $\mathrm{kWh}$ & $\%$ & million $\mathrm{kWh}$ & $\%$ \\
\hline TPP and CHP, of which: & 24132.9 & 32.9 & 23011.6 & 29.6 & -1121.3 & -4.6 \\
\hline TES GK & 17579.7 & 23.9 & 17433.1 & 22.4 & -146.6 & -0.8 \\
\hline $\mathrm{CHP}$ and cogeneration units & 6553.2 & 8.9 & 5578.5 & 7.2 & -974.7 & -14.9 \\
\hline HPPs and PSPs, of which: & 3606.4 & 4.9 & 6198.0 & 8.0 & 2591.6 & 71.9 \\
\hline НPP & 2865.5 & 3.9 & 5630.9 & 7.2 & 2765.4 & 96.5 \\
\hline PSP & 740.9 & 1.0 & 567.1 & 0.7 & -173.8 & -23.5 \\
\hline NPP & 39389.1 & 53.6 & 42004.9 & 54.0 & 2615.8 & 6.6 \\
\hline WHERE & 5356.2 & 7.3 & 5654.7 & 7.3 & 298.5 & 5.6 \\
\hline Block stations & 947.4 & 1.3 & 865.9 & 1.1 & -81.5 & -8.6 \\
\hline Total & 73432.0 & 100.0 & 77735.1 & 100.0 & 4303.1 & 5.9 \\
\hline
\end{tabular}


Table 9. The actual electricity balance of the UES of Ukraine in June 2021

\begin{tabular}{|c|c|c|c|c|}
\hline \multirow{2}{*}{ Indexes } & \multirow{2}{*}{ Forecast } & \multirow{2}{*}{ Fact } & \multicolumn{2}{|c|}{ Deviation, $+/-$} \\
\hline & & & million kWh & $\%$ \\
\hline 1. Electricity generation including: & 11599.0 & 11324.0 & -275.0 & -2.4 \\
\hline 1.1. TES & 2848.0 & 2315.0 & -533.0 & -18.7 \\
\hline 1.2. $\mathrm{CHP}$ and cogeneration units & 631.0 & 280.0 & -351.0 & -55.6 \\
\hline 1.3. HPP & 821.0 & 1200.0 & 379.0 & 46.2 \\
\hline 1.4. PSP & 143.0 & 83.0 & -60.0 & -42.0 \\
\hline 1.5. NPP & 5886.0 & 6204.0 & 318.0 & 5.4 \\
\hline 1.6. Block stations & 140.0 & 174.0 & 34.0 & 24.3 \\
\hline 1.7. Alternative sources & 1130.0 & 1068.0 & -62.0 & -5.5 \\
\hline 2. Import of electricity & 84.0 & 4.0 & -80.0 & -95.2 \\
\hline 3. Export of electricity & 485.0 & 336.0 & -149.0 & -30.7 \\
\hline $\begin{array}{l}\text { 4. Technological flow of electricity due to parallel } \\
\text { work with power systems of neighboring countries }\end{array}$ & 0 & -22 & -22.0 & \\
\hline 5. Electricity consumption (gross) & 11000.0 & 10970.0 & -30.0 & -0.3 \\
\hline 6. PSP power consumption in pump mode & 198.0 & 112.0 & -86.0 & -43.4 \\
\hline
\end{tabular}

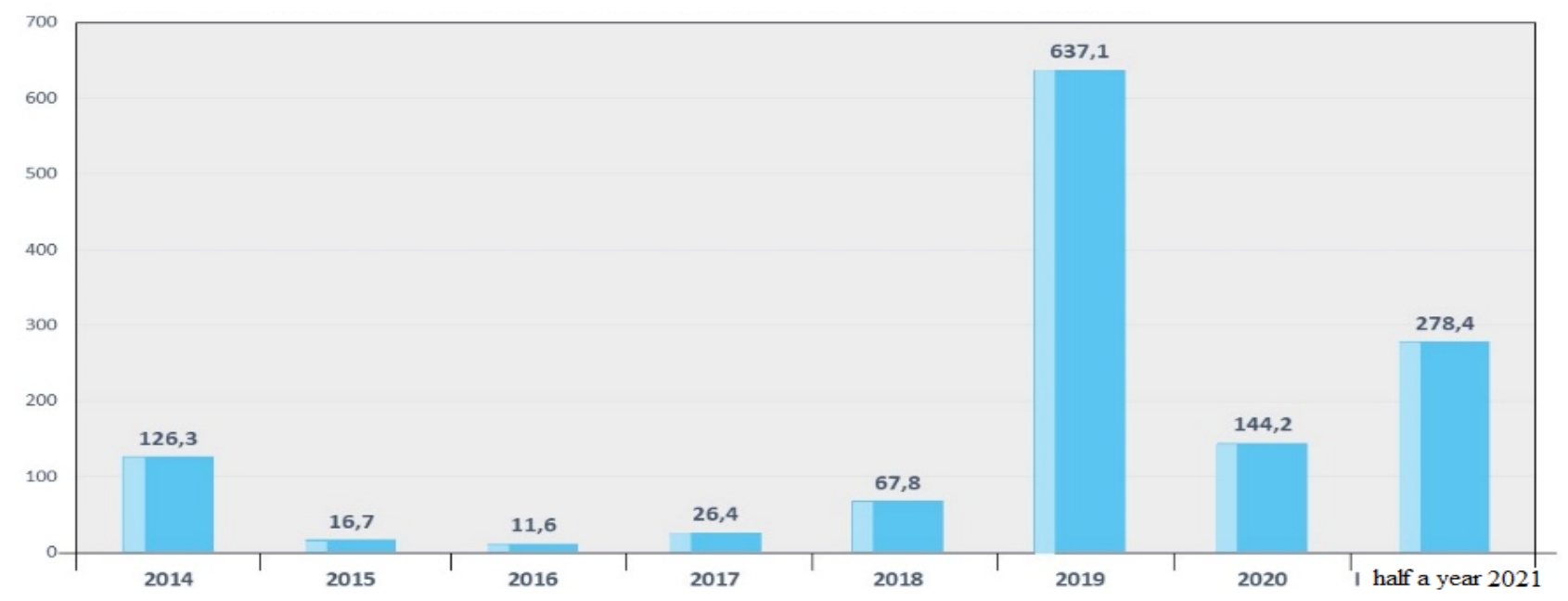

Figure 3. Annual increase in wind power capacity, 2014 - half year 2021, MW

For 6 months of 2021, the volume of electricity production by power plants of Ukraine amounted to $77,735.1$ million $\mathrm{kWh}$, which is $4,303.1$ million $\mathrm{kWh}$, or $5.9 \%$ more than for 6 months of 2020 [23].

The main share in the total production for 6 months of 2021 is the production of NPPs $-54.0 \%$, TPPs and CHPs - 29.6\%, and the production of HPPs and PSPs $-8.0 \%$. For 6 months of 2020, the share of production of NPPs, TPPs and CHPs and HPPs and PSPs was 53.6\%, 32.9\% and 4.9\%, respectively. Data on electricity production are given in Table 8. Annual increase in wind power capacity Fig. 3

In June 2021, the volume of electricity production by power plants that are part of the UES of Ukraine amounted to 11,324.0 million $\mathrm{kWh}$ and increased by 462.0 million $\mathrm{kWh}$, or $4.3 \%$ compared to June 2020 . The actual balance of electricity is given in Table 9 and the forecast for 2020-2021 in Fig. 4. UES electricity production Fig. 5 .

According to the approved forecast balance of electricity of the UES of Ukraine for 2021, the total production for this year is 151.023 billion $\mathrm{kWh}$. This is $5.5 \%$ (7.933 billion $\mathrm{kWh})$ more than projected in the forecast balance for 2020 .
Generations:

- NPP: production of "nuclear" electricity for the next year is determined taking into account the proposals of NNEGC "Energoatom" at the level of 75.3 billion $\mathrm{kWh}$, which is 1.5 billion kWh more than forecast in 2020. Next year's NPP production will traditionally occupy a dominant share in Ukraine's UES $50 \%$;

- TPP: electricity generation at TPPs is planned at 41.140 billion $\mathrm{kWh}$ or $27 \%$ of the total;

- CHP: production volumes of CHPs and cogeneration units, taking into account their thermal schedule and taking into account the actual supply of electricity during the current year, are set at 12.790 billion $\mathrm{kWh}$ or about $8 \%$ of the total;

- HPPs and PSPs (including HPPs at the "green" tariff): the projected volumes at HPPs ( 5.287 billion $\mathrm{kWh}$ ) are determined taking into account the low water content of the year in recent years. PSP power generation is set at 1.7 billion $\mathrm{kWh}$. In total, about $5 \%$ of the total; 


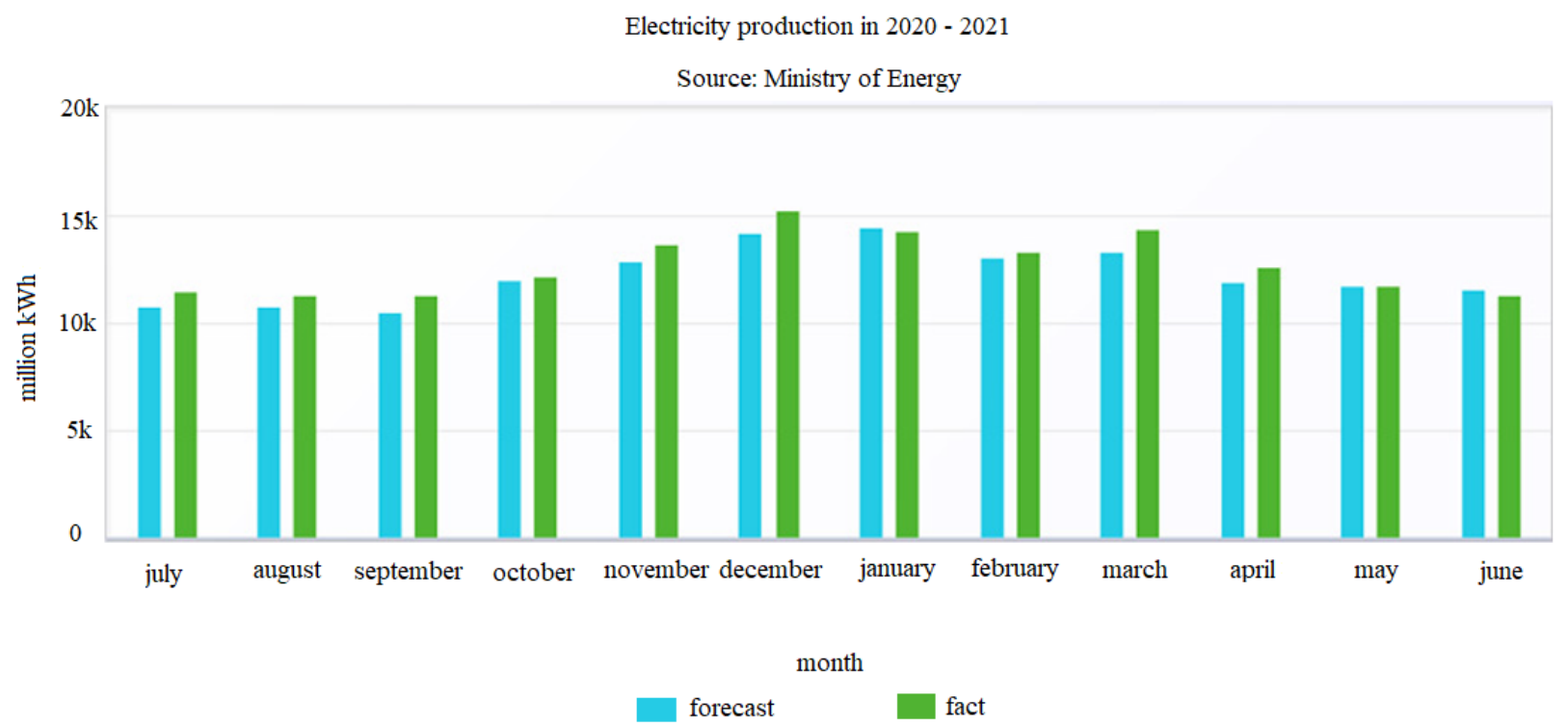

Figure 4. Forecast of electricity production 2020-2021

Projected volumes of electricity production of UES of Ukraine for 2021

billion $\mathrm{kWh}$

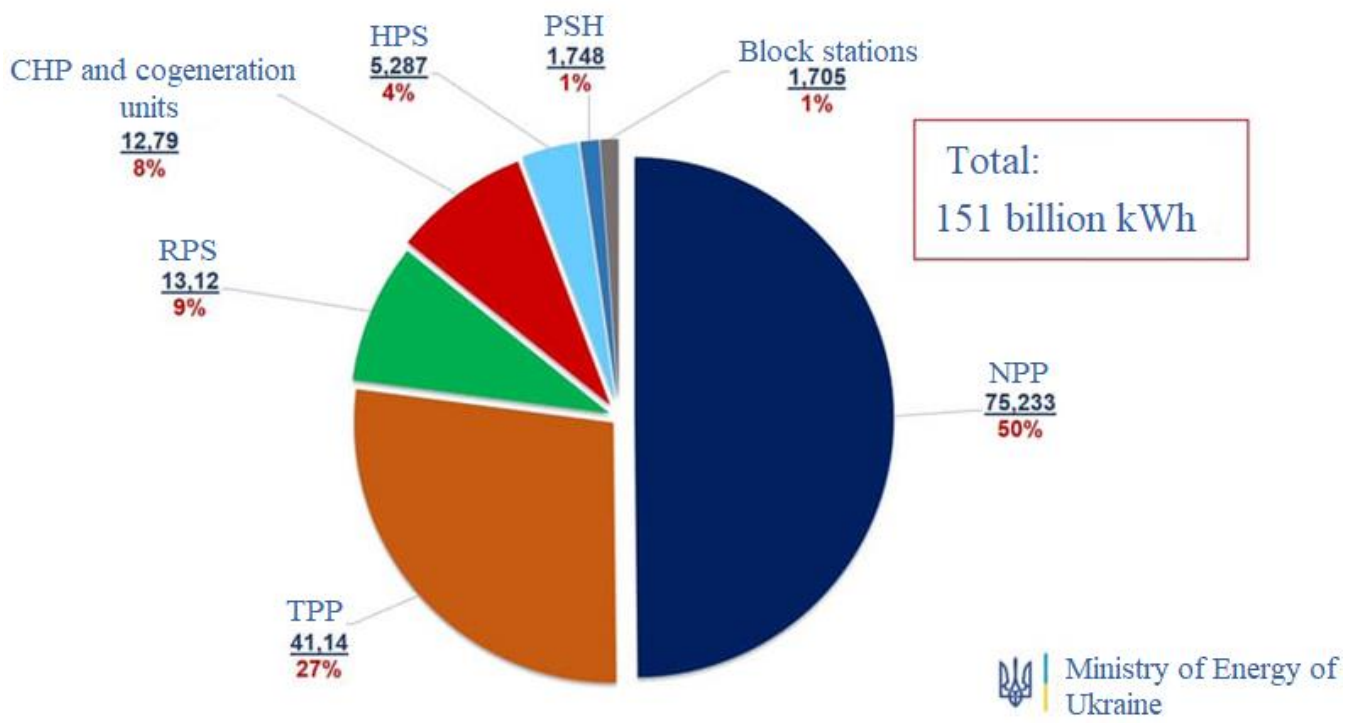

Figure 5. UES electricity production for 2021

- RES: the projected balance of the volume of electricity production from alternative sources - 13.12 billion $\mathrm{kWh}$. take into account possible limitations of SES and WPP generation at the level of 1.5 billion $\mathrm{kWh}$. In total, about $9 \%$ of the total;

- Block stations: 1.705 billion $\mathrm{kWh}$ - about $1 \%$.

The actual balance of the UES for 2021 shown in Fig. 6.

\section{MATHEMATICAL METHOD}

The energy sector is constantly evolving, and significant inventions and innovations are still ahead. Energy consumption has always been involved in other industries, such as agriculture, manufacturing, transportation and many others. Thus, these industries tend to increase the amount of energy they consume daily. Energy seems very demanding in terms of the application of new technologies and the development of new energy sources.

The rapid development of the energy sector and utilities directly affects social development. People now face the challenges of sound management and energy consumption, the use of renewable energy sources and the protection of the environment. Smart technologies play a crucial role in addressing these issues. In this article, we will consider the most striking cases of the use of scientific data in the field of energy and utilities. 


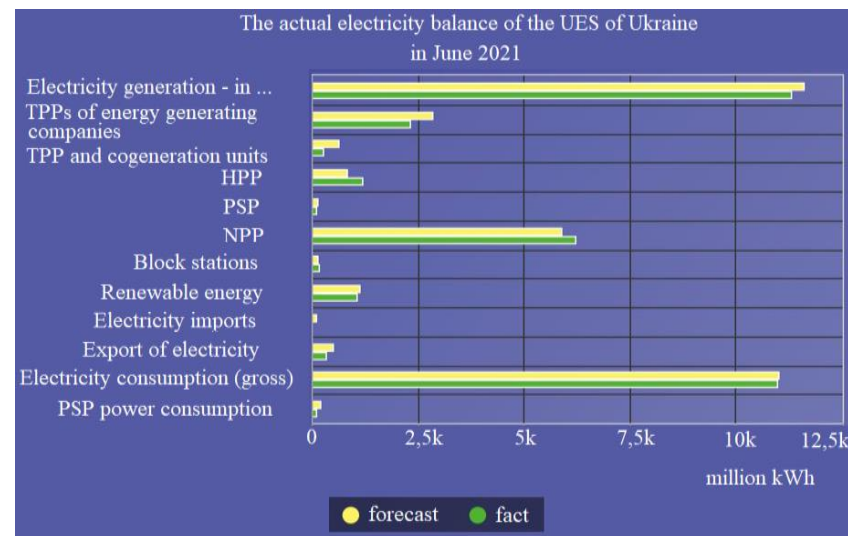

Figure 6. The actual electricity balance of the UES for 2021

\section{Failure Probability Modeling}

Failure probability modeling has gained its place in the energy sector. The effectiveness of machine learning algorithms in predicting failures, no doubt.

Active application of probability modeling helps to increase productivity, anticipate accidental malfunctions and, as a result, reduce maintenance costs. Energy companies invest heavily in the maintenance and proper operation of their machines and devices. Unexpected failures in their activities lead to significant financial losses. Moreover, for people who rely on these companies as a source of energy, the situation becomes critical. As a result, the overall reliability and image of the energy supplier may be affected [8].

\section{FAUlt DeteCtion AND ForeCASTING}

Despite the efforts of energy companies, power outages are still occurring, with a significant number of people running out of energy. In this regard, people tend to view power outages as a malfunction of electrical networks. However, de-energization is a precautionary measure, the result of an automatic protection system.

In previous years, energy system engineers used static algorithms and models instead of real-time solutions. Numerous energy and utility companies are currently actively upgrading their systems to improve outage detection and prediction. Modern intelligent communication systems with power outages are capable of: forecasting the impact of weather conditions on the grid forecasting the impact of the value of short-term assets on the grid detection of possible outages using intelligent events and communication on this subject [24].

Fault detection and prediction begins with identifying the correct metrics and the threshold for them. Each individual case of failure must be carefully analyzed to identify the root cause. Only then can predictive algorithms be used to model the future probability of tripping. The use of intelligent power outage ecosystems allows for the provision of accurate power outage statuses in real time to improve the overall experience and customer satisfaction.

Dynamic energy management systems belong to an innovative approach to load management. This type of management covers all traditional principles of energy management related to demand, distributed energy sources and demand management, as well as current energy issues such as energy savings, temporary load and demand reduction. Thus, smart energy management systems have developed the ability to combine smart end-use devices, distributed energy resources and improved management and communication [25].

Big data analytics plays a leading role here, as it provides the capabilities of dynamic control systems in Smart Grids. This greatly helps to optimize energy flows between suppliers and consumers. The efficiency of the energy management system, in turn, depends on load forecasting and renewable energy sources.

The dynamic energy management component typically includes smart energy end-use devices, intelligently distributed power sources, advanced control systems, and an integrated communication architecture.

Dynamic energy management systems process a huge amount of data obtained by practical methods and solutions. Applying big data analytics to this data helps to assess performance and provide sound recommendations for energy management [26].

The development of methods for optimizing small energy complexes, which is not tied to a specific consumer or a fixed composition of equipment, involves the formation of a unified approach to mathematical modeling of various installations that can be included in the energy complex [27].

In addition, the choice of optimization method and its adaptation to solve the problem is preceded by its formalization and selection of optimization criteria.

Formalization of the problem involves the compilation of a generalized mathematical model of the energy complex of arbitrary composition, as well as the formation of a system of assumptions that simplify the calculations.

The task of the energy complex is uninterrupted energy supply specified consumer. That is, at any time, the thermal and electrical load of the energy consumer must be fully provided. In the general case, in addition to the energy produced by the generating units, the consumer may receive heat or electricity from the centralized power supply networks or use the energy stored in the batteries [21].

The mathematical model of the energy complex of arbitrary composition is based on the equations of the energy balance of heat and electricity flows: 


$$
\begin{aligned}
& Q_{e c}^{j}=Q_{e g}^{j}+\sum_{i=1}^{m_{Q}} Q_{i}^{j}, \\
& N_{e c}^{j}=N_{e g}^{j}+\sum_{i=1}^{m_{N}} N_{i}^{j},
\end{aligned}
$$

where $Q_{e c}^{j}, N_{e c}^{j}$ are the thermal and electrical load of the consumer on the $j$-th time interval; $Q_{e g}^{j}, N_{e g}^{j}$ are the thermal and electric power, characterize the interaction of the consumer with external energy networks (these values are positive if the power is imported from external networks and negative if the power is exported); $m_{Q}, m_{N}$ are the number of heat and power plants in the energy complex, respectively; $Q_{i}^{j}$ is the thermal capacity of the $i$-th installation on the $j$-th time interval; $N_{i}^{j}$ is the electric power of the $i$-th installation on the $j$-th time interval. If the installation does not produce but consumes the appropriate type of energy (for example, electric boilers or batteries), these values may be negative. If the energy complex exports energy to external networks, the values $Q_{e g}^{j}$ will also be negative.

The generalized scheme of the complex of small distributed energy connected to external power networks, with indication of directions of streams of thermal and electric energy is presented on Fig. 7.

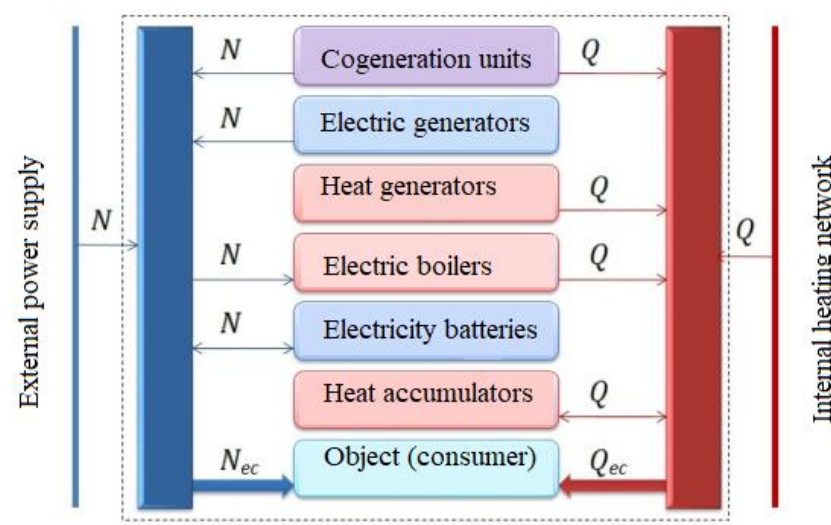

Figure 7. Generalized scheme of the energy complex

As a parameter that characterizes the mode of operation of power plants, we introduce the load factor, which is calculated as the ratio of the current capacity of the plant to its nominal value

$x_{i}^{j}=\frac{P_{i}^{j}}{P_{i}^{\text {nom }}}$,

where $x_{i}^{j}$ is the load factor of the $i$-th installation on the $j$-th time intervals; $P_{i}^{j}$ is the power of the $i$-th installation on the $j$-th time interval; $P_{i}^{n o m}$ is the nominal value of power of the $i$-th installation. $P_{i}^{n o m}$ is determined for electric and heat generating installations.

For cogeneration units, the current electric and rated electric power, respectively, are selected as $P_{i}^{j}$ and $P_{i}^{n o m}$. The parameter $x_{i}^{j}$ at any time determines for a particular installation its performance and associated fuel costs. This is an adjustable parameter, which means an optimization parameter.

Nominal power of power plants is also an optimization parameter in solving the problem of finding the optimal configuration of equipment. If the nominal capacity is the same for the entire calculation period, the load factor of each installation is determined for each of the time segments that make up the calculation horizon, ie, in the case of energy systems - 1 year. The choice of such a calculation horizon is due to the fact that the load of the consumer and the climatic conditions that determine the mode of operation of RES installations have exactly this frequency.

The year is divided into hours, within which the consumer's load is considered constant, which means that there are 8760-time intervals within the calculation period.

When solving the problem of finding the optimal mode of operation for the power complex, including 4 generating units, the number of optimized parameters will be $4 \times 8760=35040$. In fact, the number of variables can be much larger, and when finding the optimal configuration, the task is much more difficult work of installations which can not enter into the optimum structure of the equipment in general.

Methods of linear programming allow to solve quite successfully problems of optimization of the systems characterized by a large number of variables.

The problem of linear programming is formulated as follows: to find a vector of values of variables that deliver the extremum of a linear objective function under given constraints in the form of linear equalities or inequalities. One of the most effective methods of linear programming is the simplex method. This method implements a rational search of basic admissible solutions in the form of a final iterative process, improves the value of the objective function (minimum optimization criterion) at each step establish that there is no optimal solution [28].

When choosing a simplex method as an optimization method, it is necessary that the mathematical model of the object used satisfies the constraints imposed by the method, namely:

1) mathematical models of individual installations and the imposed restrictions are always linear; 
2) any variables used in solving problems by the simplex method must be non-negative. This means that if any installation, such as an electric boiler, consumes energy, then its contribution to the balance is written with a sign " $-"$. If the installation can both consume and give energy to the consumer (batteries), then its participation in the energy balance will be characterized by two variables, one of which will be non-zero if the installation produces energy, and the other - non-zero if the installation consumes energy.

Also, in order to simplify the calculations and models used, the following assumptions are introduced:

1) temporal changes in the characteristics of the installations associated with the start, stop and transient modes are not taken into account, because the transient modes occupy a small proportion of the operating time of the installations;

2) small changes in the characteristics of installations as a result of changes in the mode of their operation are not taken into account, because the impact of these changes on the overall efficiency of the system is negligible. For example, the efficiency of electric boilers changes during operation within $5 \%$, and the nominal value of the efficiency of different types of electric boilers is from $90 \%$ to $98 \%$;

3) batteries at the beginning of the billing period in full discharged;

4) several batteries of the same type of energy are replaced when calculating one battery of total capacity.

When creating the simplest, "Naïve" forecasting models assume that some recent period of the forecast time series best describes the future of this forecast series, so in these models the forecast is usually a very simple function of the values of the forecast variable in the recent past [29]. The simplest model is

$Y(t+1)=Y(t)$,

which corresponds to the assumption that "tomorrow will be like today". Obviously, one should not expect great accuracy from such a primitive model. This model can be slightly improved as follows:

$$
\begin{aligned}
& Y(t+1)=Y(t)+[Y(t)-Y(t-1)], \\
& Y(t+1)=Y(t) \times[Y(t)-Y(t-1)] .
\end{aligned}
$$

In this way, adapt the model to possible trends. There are also approaches in building simple models, which are to use average readings. The model, based on simple averaging, looks like this:

$$
Y(t+1)=(1 / t) \times[Y(t)+Y(t-1)+\ldots+Y(1)] .
$$

This model corresponds to the principle "tomorrow will be as it was on average recently". This model, of course, is more resistant to fluctuations, because it smooths out random emissions relative to the average. Despite this, this method is ideologically as primitive as the "naïve" models, and it has almost the same shortcomings.

When forecasting, the method of exponential averages is often used, which is constantly adapting to the data due to new values. An expression describing this model:

$$
Y(t+1)=a \times Y(t)+(1-a) \times Y^{\prime}(t),
$$

where $Y(t+1)$ is the forecast for the next period of time; $Y(t)$ is the real value at time $t ; Y^{\prime}(t)$ is the past forecast at time $t ; a$ is the smoothing constant $(0 \leq a \leq 1)$.

The internal parameter $a$ determines the dependence of the forecast on older data, and the impact of data on the forecast decreases exponentially with the "age" of the data. It is obvious that for $a \rightarrow 1$ the exponential model tends to the simplest "naïve" model, and for $a \rightarrow 0$ the predicted value becomes equal to with a preliminary forecast.

Although the models described above are sometimes used in forecasting in not very difficult situations, it is still not recommended to use them in real forecasting problems due to the obvious primitiveness and inadequacy of the models.

In all this, the described algorithms can be successfully used as ancillary and auxiliary for data processing in forecasting problems.

For example, in most cases it is necessary to decompose time series (separate trend, seasonal and irregular components). One of the methods of selecting trend components is the use of exponential smoothing.

However, there are more adequate forecasting methods. One of them was proposed to Holt in the middle of the last century. It is an improved method of exponential smoothing, later named after the scientist [30].

In the proposed algorithm, the values of the level and trend are smoothed using exponential smoothing, with different smoothing parameters:

$\left\{\begin{array}{l}\Omega_{t}=\alpha Y_{t}+(1-\alpha)\left(\Omega_{t-1}-T_{t-1}\right), \\ T_{t}=\beta\left(\Omega_{t}-\Omega_{t-1}\right)+(1-\beta) T_{t-1}, \\ Y_{t+p}^{\prime}=\Omega_{t}+\rho T_{t} .\end{array}\right.$

Here, the first equation describes a smoothed series of general levels. The second equation is used to estimate the trend. The third equation determines the prediction for $p$ samples over time.

Constant smoothing in the Holt method ideologically plays the same role as the constant in simple exponential smoothing. This pair is selected in prac- 
tice so that the model gives the greatest accuracy on a test set.

The main disadvantage of the Holt method is that it does not take into account seasonal fluctuations in forecasting. In other words, this method cannot "see" them in prehistory.

There is an extension of the Holt method to riparametric exponential smoothing - the Winters method. An attempt is made to take into account seasonal components in the data. The system of equations describing the Winters method is as follows:

$$
\left\{\begin{array}{l}
\Omega_{t}=\alpha Y_{t}+(1-\alpha)\left(\Omega_{t-1}-T_{t-1}\right), \\
T_{t}=\beta\left(\Omega_{t}-\Omega_{t-1}\right)+(1-\beta) T_{t-1}, \\
S_{t}=\gamma \frac{Y_{t}}{\Omega_{t}}+(1-\gamma) S_{t-s}, \\
Y_{t+p}^{\prime}=\left(\Omega_{t}+\rho T_{t}\right) S_{t-s+p} .
\end{array}\right.
$$

The fraction in the first equation is used to exclude seasonality with $Y_{t}$ after excluding seasonality, the algorithm works with "pure" data in which there are no seasonal fluctuations.

They appear in the final forecast, when the "pure" forecast, calculated almost by the method of Holt, is multiplied by the seasonal factor.

Along with methods based on exponential smoothing, regression algorithms have been used for prediction for a long time. Briefly, the essence of algorithms of this class can be described as follows.

There is a predicted variable $Y$ (dependent variable) and selected in advance a set of variables on which it depends $-X_{1}, X_{2}, \ldots, X_{N}$ (independent variables). The nature of the independent variables may be different. Then the multiple regression model is generally described by the expression

$Y=F\left(X_{1}, X_{2}, \ldots, X_{N}\right)+\varepsilon$

In a simpler version of the linear regression model, the dependence of the variable has the form:

$Y=\beta_{0}+\beta_{1} X_{1}+\beta_{2} X_{2}+\ldots+\beta_{N} X_{N}+\varepsilon$,

where $\beta_{0}, \beta_{1}, \beta_{2}, \ldots, \beta_{N}$ are selected regression coefficients; $\varepsilon$ is the error component.

To build regression models, it is necessary to have a database, an example of which is presented in the following Table 10.

Table 10. Observation database

\begin{tabular}{cccccc}
\hline \multirow{2}{*}{ No. } & \multicolumn{5}{c}{ Variables } \\
\cline { 2 - 6 } & $X_{1}$ & $X_{2}$ & $\ldots$ & $X_{N}$ & dependent \\
\hline 1 & $X_{11}$ & $X_{12}$ & $\ldots$ & $X_{1 N}$ & $Y$ \\
2 & $X_{21}$ & $X_{22}$ & $\ldots$ & $X_{2 N}$ & $Y_{2}$ \\
$\ldots$ & $\ldots$ & $\ldots$ & $\ldots$ & $\ldots$ & $\ldots$ \\
$m$ & $X_{m 1}$ & $X_{m 2}$ & $\ldots$ & $X_{m N}$ & $Y_{m}$ \\
\hline
\end{tabular}

Using the table of values of past observations, regression coefficients are selected (for example, by the method of least squares), and thus the model is adjusted.

Neural network (artificial neural network) mathematical model, as well as its software or hardware embodiment, built on the principle of organization and functioning of biological neural networks - networks of nerve cells of a living organism [31]. In Fig. 8 shown an example of a neural network.

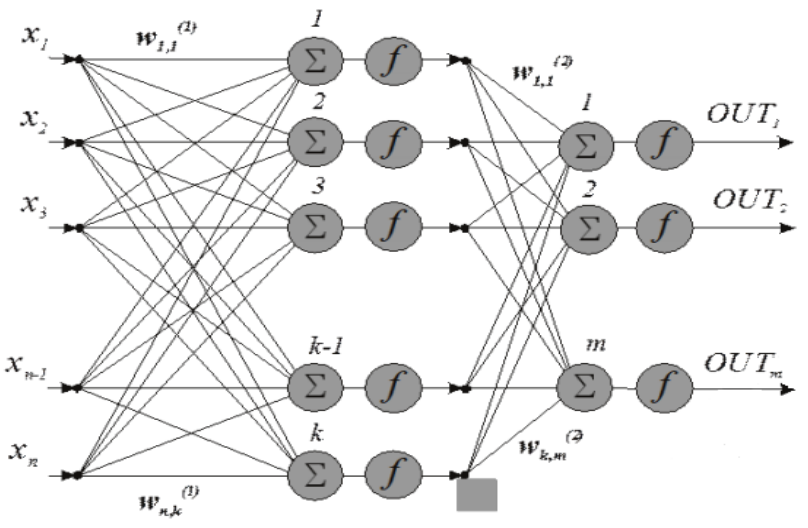

Figure 8. Example of a neural network

This type of algorithms, built on neural network technologies, has several significant advantages:

- effective work with dynamic systems;

- the possibility of setting a not fully formalized task;

- high failure resistance;

- the ability to select patterns in a large amount of information, etc.

All the data of dignity have become a driver for the widespread use of these algorithms and their application in economics, medicine, robotics, information technology, etc.

Currently, according to many authors and researchers, a promising quantitative method of forecasting is the use of neural networks. The reason for this is the serious advantages of the neural network approach. Consider the most important of them.

When using neural networks, it is easy to investigate the dependence of the predicted value on independent variables. for example, there is an assumption that the electrical load next week somehow depends on the following parameters:

- load in the last week;

- load in the penultimate week;

- number of working days;

- length of daylight;

- weather temperatures.

At the same time, it is necessary to build a system that would include all the parameters of natural factors and would build short-term forecasts. 
In this formulation of the problem, most of the classical methods of forecasting will simply fail. The use of even the simplest neural network architecture (Perceptron with one hidden layer) and database (with electrical load and all parameters) easily gives a working forecasting system. And the account or disregard of system of external parameters will be defined by enabling or disabling the corresponding input to the neural network.

Another major advantage of neural networks is that the expert is not hostage to the choice of mathematical model time series behavior. The construction of a neural network model is adaptive during training, without the participation of an expert. In this case, the neural network presents examples from the database and it itself adapts to this data.

In addition, neural networks have the ability to adapt to change environment. In particular, neural networks trained to operate in a particular environment can be easily retrained to operate under conditions of small fluctuations in environmental parameters. Moreover, to work in a non-stationary environment, neural networks can be created that change their weather forecasts in real time.

The uniformity of neural network analysis and design is also important. This means that the same neural network design solution can be used in many subject areas. It should be noted, however, that neural networks have inherent shortcomings, one of which is their non-determinacy. It is implied that after training the network is a "black box" [32], which somehow works, but the logic of decisionmaking by the neural network is completely hidden from the expert. In principle, there are algorithms for "extracting knowledge from the neural network", which formalize the learned neural network to a list of logical rules, thereby creating an expert system based on the network.

Thus, of the considered forecasting methods, the neural network approach looks the most adequate, accurate and universal. On the other hand, its adaptation to specific practical tasks and analysis of forecasting results, of course, is of scientific interest.

Data Science is the science of data, combining different fields of knowledge: computer science, mathematics and systems analysis. These include methods of big data processing (Big Data), data mining (Data Mining), statistical methods, methods of artificial intelligence, including machine learning (Machine Learning) [18]. DS includes methods of designing and developing databases and application software [31].

The main practical purpose of the work is to extract useful information from large arrays of information, identify patterns, develop and testing hypotheses by modeling and developing new software.

To achieve this goal, Data Scientist uses the following tools [33]:
- statistical modeling packages (R-Studio, Matlab);

- Big Data technologies (Apache Hadoop, HDFS, Spark, Kafka), NoSQL-DBMS (Cassandra, HBase, MongoDB, DynamoDB and other non-relational solutions);

- SQL for work with classical relational databases and formation of structured queries to NoSQL-solutions by means of Apache Phoenix, Drill, Impala, Hive, etc.;

- programming languages (Python, R, Java, Scala) for the development of machine learning models and software prototypes;

- information systems of the Business Intelligence class (dashboards, data showcases) for visualization of business indicators from information arrays.

Thus, we can conclude that Data Science includes the following areas of knowledge:

- mathematics: mathematical analysis, mathematics and mathematics;

- informatics: development of software, databases, models and algorithms of machine learning (neural networks, Bayesian algorithms, regression series, etc.), Data Mining;

- system analysis (methods of subject area analysis, Business Intelligence).

In energy, Data Science can help solve many problems, such as forecasting electricity consumption, forecasting electricity prices, calculating optimal tariffs, diagnosing energy facilities, optimizing consumption patterns and more.

Analytical calculations of processes in power supply systems [34], the key prerequisite is the introduction of efficient control algorithms for MicroGrid systems, process modeling and predictive capabilities for more flexible management of local power systems. Analytical methods are most suitable for this purpose, which, in contrast to numerical ones, allow the analysis of a nonlinear circuit in general, and not for certain values of the parameters of the circuit elements.

As an example, consider the method of individual components using Laplace transforms. The vast majority of complex periodic functions, which describe the modes of operation of electricity generators, can be formed from elementary mathematical functions, which are called basic (Table 11).

However, the approximation of the nonlinear characteristic that underlies these methods, leads to the introduction of calculations of greater or lesser error.

The image $F_{n}(p)$ of the signal function $U(t)$ for the case of rectangular pulses with amplitude $U_{i}$ has the form: 
Table 11. Basic composition of complex periodic functions

\begin{tabular}{clcc}
\hline Type & \multicolumn{1}{c}{ Name } & Basic function $f B$, and $(t)$ & $F B$, and $(t)$ \\
\hline 1 & Level function & $f B, 1(t)=A$ & $F B, 1(t)=\frac{A}{p}$ \\
2 & Sinusoidal function & $f B, 2(t)=\sin \omega t$ & $F B, 2(t)=\frac{\omega}{\left(p^{2}+\omega^{2}\right)}$ \\
3 & Exponential function & $f B, 3(t)=e^{-\sigma t}$ & $F B, 3(t)=\frac{1}{(p+\alpha)}$ \\
4 & Direct proportionality function & $f B, 4(t)=A t$ & $F B, 4(t)=\frac{A}{p^{2}}$ \\
\hline
\end{tabular}

$F_{n}(p)=\frac{1}{p} \frac{\left(1-e^{-p T}\right)}{\left(1-e^{-p T_{n}}\right)} \sum_{i=1}^{n} U_{i} e^{-(i-1) p T}$.

When the intervals of approximation of the function have different durations, namely $=$ var, $T_{i}$ which is a more generalized case, the image of the signal function is as follows:

$$
F_{n}(p)=\frac{1}{p} \frac{1}{\left(1-e^{-p T_{n}}\right)} \sum_{i=1}^{n} U_{i}\left(e^{-p T_{(i-1)}}-e^{-p T_{i}}\right) .
$$

provided that $T_{n}=\sum_{i=1}^{n} T_{i}, T_{0}=0$.

Calculations according to (13) in comparison with (14) are more difficult, however in some cases implementation of approximation of function of a signal of the generator can simplify calculations, having reduced quantity of intervals of approximation at the expense of combination of intervals with identical amplitudes of step pulses [35].

When constructing modulated functions and signal functions, for the approximation of which it is necessary to apply several basic functions, it is necessary to use the alternation of the basic function with a pause to form a complex basic function. In this case $T_{i}+T_{0 i}=T_{n}$, where $T_{i}$ is the duration of the basic function, $T_{0 i}$ is the duration of the pause. With:

$F_{i}(0+t) \equiv F_{i}\left(T_{i}+T_{0 i}+t\right)$.

Then the image function of the signal is as follows:

$F_{n}(p)=\frac{1}{p} \frac{\left(1-e^{-p T}\right)}{\left(1-e^{-p T_{n}}\right)} \sum_{i=1}^{n} U_{i} e^{-2(i-1) p T}$.

The shift of the whole function is carried out by the delay theorem by multiplying by. It should be noted that when approximating several basic functions, it is necessary to comply with the requirements: where, the requirement of unambiguous definition of the image of the function on the interval $e^{-p T} F_{i}\left(f_{e i}, T_{i}\right) \cap F_{j}\left(f_{e j}, T_{j}\right)=\varnothing \quad, \quad i \neq j \quad$ and $i, j \in[1, \ldots, n]$.
The sequence of calculations for finding generalized expressions for calculations of processes in circles with cyclically variable parameters is as follows:

1) construction of graphs or tables of dependences of cyclic changes of parameters of an electric circuit on time, in the same scale, definition of functions of the generator $U_{g}(t)$ and loading $Z(t)$;

2) construction of images of the functions of the generator $U(p)$ and the load $Z(p)$ using Laplace transforms: $\quad U(p)=L\left\{U_{g}(t)\right\} Z(p)=$ $=L\{Z(t)\}$;

3) construction of images of current functions in accordance with Ohm's law in operator form: $I(p)=U(p) / Z(p)$;

4) finding the original currents using the inverse Laplace transform $I(t)=L^{(-1)}\{I(p)\}$.

Obtaining current functions at intervals in analytical form allows accurate calculations to assess current characteristics, optimal choice of parameters of electronic and electrical devices, it is necessary to determine a number of characteristics and coefficients, including current and average currents, amplitude maxima and minima, shape coefficients, pulsation amplitudes value, ripple at the current value, ripple at the average value, amplitude.

In turn, this makes it possible to model and forecast the operation of a complex power supply system of the territory with several power generators and complex loads, both to optimize the current operation of such a system and to improve and plan development.

Regression analysis is a set of statistical methods for studying the effect of one or more independent variables $X_{1}, X_{2}, \ldots, X_{p}$ on the dependent variable $Y$. Independent variables are otherwise called regressors or predictors, and dependent variables - criterion. The terminology of dependent and independent variables reflects only the mathematical dependence of variables, not causal relationships. The most common type of regression analysis is linear regression, when a linear function is found 
that, according to certain mathematical criteria, best meets the data. For example, the least squares method calculates a line (or hyperplane), the sum of the squares between which and the data is minimal. Objectives of regression analysis:

- determination of the degree of determinism of variation of the criterion (dependent) variable by predictors (independent variables);

- predicting the value of a dependent variable using an independent one;

- determining the contribution of individual independent variables to the variation of the dependent one.

\section{CONCLUSION}

The process of implementation of Smart Grid technologies for the Ukrainian power industry will cause the following fundamental changes in comparison with the current state of the power system:

- transition from centralized methods of generation and transmission of electricity to distributed with the ability to manage energy production facilities and network topology at any point, including at the consumer level;

- replacement of centralized demand forecasting by the methodology of active consumer influence, which becomes an element and subject of the management system;

- abandonment of strict dispatching regulation in favor of coordinating the work of all components of the network;

- transfer to Smart-technology of processes of control, accounting and diagnostics of assets that will provide perspective opportunities of self-restoration of power system, and also an effective mode of operation of fixed assets;

- construction of high-performance information and computing infrastructure as the core of the energy system;

- formation of preconditions for wide introduction of the new devices increasing maneuverability and controllability of the equipment

- flexible connections, DC inserts, energy storage devices, etc.;

- development of distributed "intelligent" management systems and analytical tools to support the development and implementation of real-time solutions;

- creation of next-generation operational applications (SCADA/EMS/NMS) that allow the use of innovative algorithms and methods of power system management, including its new active elements.

And with the help of tools: python, data science, big data, machine learning, AI, the process of
Ukraine's transition to a new level of energy will be quite powerful.

So on the basis of these features can be given a fairly accurate definition of "intelligent" electrical network, as a set of connected to the generating sources and electrical installations of consumers of software, as well as information-analytical and control systems that provide reliable and high-quality transmission of electricity from source to receiver, the right time and in the right amount.

\section{DISCLOSURE STATEMENT}

No potential conflict of interest was reported by the author(s).

\section{REFERENCES}

1. Vesnic-Alujevic, L., Breitegger, M., \& Pereira, A.G. (2016). What smart grids tell about innovation narratives in the European Union: Hopes, imaginaries and policy. Energy Research \& Social Science, 12, 16-26. https://doi. org/10.1016/j.erss.2015.11.011

2. Panda, D.K., \& Das, S. (2019). Regression analysis of grid stability under decentralized control. In 2019 International Conference on Engineering, Science, and Industrial Applications (ICESI) (pp. 1-6). IEEE. https://doi.org/10. 1109/ICESI.2019.8863027

3. Tan, S.O., Türker, İ., \& Toku, T. (2017). The scientific studies on smart grid in selected European countries. MATEC Web of Conferences, 112, 10012. https:/ / doi.org/10. 1051/matecconf/201711210012

4. Wang, C., Lv, T., \& Deng, X. (2020). Bibliometric and visualized analysis of China's smart grid research 2008-2018. Frontiers in Research Metrics and Analytics, 5, 551147. https:/ / doi.org/10.3389/frma.2020.551147

5. Leal-Arcas, R., Lesniewska, F., \& Proedrou, F. (2018). Smart grids in the European Union: Assessing energy security, regulation \& social and ethical considerations. Columbia Journal of European Law, 24(2), 291-389. https: / / ssrn.com/abstract=3062957

6. Butt, O.M., Zulqarnain, M., \& Butt, T.M. (2021). Recent advancement in smart grid technology: Future prospects in the electrical power network. Ain Shams Engineering Journal, 12(1), 687-695. https://doi.org/10. 1016/j.asej.2020.05.004

7. Pašalić, I.N., Ćukušić, M., \& Jadrić, M. (2021). Smart city research advances in Southeast Europe. International Journal of Information Management, 58, 102127. https://doi.org/10.1016/j.ijinfomgt.2020.102127

8. Schneider, J., Dziubany, M., Schmeink, A., Dartmann, G., Gollmer, K.U., \& Naumann, S. (2019). Predicting energy consumption using machine learning. In G. Dartmann, H. Song, \& A. Schmeink (Eds.), Big Data Analytics for Cyber-Physical Systems (pp. 167-186). Elsevier. https:/ / doi.org/10.1016/B978-0-12-816637-6.00008-7

9. Shaukat, M.A., Shaukat, H.R., Qadir, Z., Munawar, H.S., Kouzani, A.Z., \& Mahmud, M.A. (2021). Cluster analysis and model comparison using smart meter data. Sensors, 21(9), 3157. https:/ / doi.org/10.3390/s21093157 
10. Dovgalyuk, O., Omelianenko, H., Pirotti, A., Bondarenko, R., \& Syromyatnikova, T. (2019). Reliability increase of the distribution electric networks operation in the implementation of the energy market in Ukraine. In 2019 IEEE 6th International Conference on Energy Smart Systems (ESS) (pp. 70-75). IEEE. https://doi.org/10.1109/ ESS.2019.8764243

11. Bulatov, Y.N., Kryukov, A.V., \& Arsentiev, G.O. (2018). Intelligent electrical networks based on controlled energy routers. In 2018 International Conference on Industrial Engineering, Applications and Manufacturing (ICIEAM) (pp. 1-5). IEEE. https://doi.org/10.1109/ICIEAM.2018. 8728738

12. Mikhnev, I.P., Salnikova, N.A., Orudjev, N.Y., \& Shestopalova, E.L. (2020). The introduction of intelligent electrical networks in Russia. IOP Conference Series: Materials Science and Engineering, 913(5), 052032. https://doi. org/10.1088/1757-899X/913/5/052032

13. Hogselius, P., \& Kaijser, A. (2019). Energy dependence in historical perspective: The geopolitics of smaller nations. Energy Policy, 127, 438-444. https://doi.org/10. 1016/j.enpol.2018.12.025

14. Sarkar, S., Chakrabarti, U., Bhattacharyya, S., \& Chakrabarti, A. (2020). A comprehensive assessment of the need and availability of Smart Grid technologies in an electricity distribution grid network. Journal of the Institution of Engineers (India): Series B, 101, 753-761. https:/ / doi. org/10.1007/s40031-020-00486-1

15. Tominaga, T., Hachiya, M., Tatsuzaki, H., \& Akashi, M. (2014). The accident at the Fukushima Daiichi nuclear power plant in 2011. Health Physics, 106(6), 630637. https://doi.org/10.1097/HP.0000000000000093

16. Weselek, A., Ehmann, A., Zikeli, S., Lewandowski, I., Schindele, S., \& Högy, P. (2019). Agrophotovoltaic systems: applications, challenges, and opportunities. A review. Agronomy for Sustainable Development, 39(4), 35. https://doi.org/10.1007/s13593-019-0581-3

17. Seefried, A., Müller, B., \& Förster, E. (2020). Regional analysis of potentials of flexibility options in the electricity system for the study regions Prignitz in Brandenburg and Anhalt-Bitterfeld-Wittenberg in SaxonyAnhalt. Journal of Sustainable Development of Energy, Water and Environment Systems, 8(1), 162-183. https://doi.org/ 10.13044/j.sdewes.d7.0277

18. Pramangioulis, D., Atsonios, K., Nikolopoulos, N., Rakopoulos, D., Grammelis, P., \& Kakaras, E. (2019). A methodology for determination and definition of key performance indicators for smart grids development in island energy systems. Energies, 12(2), 242. https://doi. org/10.3390/en12020242

19. Pliuhin, V., \& Teterev, V. (2021). Possibility implementation analysis of the Smart Grid Network in a current state conditions of the United Energy Systems of Ukraine. Lighting Engineering \& Power Engineering, 60(1), 15-22. https:/ / doi.org/10.33042/2079-424X.2021.60.1.03

20. Otuoze, A.O., Mustafa, M.W., \& Larik, R.M. (2018). Smart grids security challenges: Classification by sources of threats. Journal of Electrical Systems and Information Technology, 5(3), 468-483. https://doi.org/10.1016/j.jesit.2018. 01.001
21. Worighi, I., Maach, A., Hafid, A., Hegazy, O., \& Van Mierlo, J. (2019). Integrating renewable energy in smart grid system: Architecture, virtualization and analysis. Sustainable Energy, Grids and Networks, 18, 100226. https:/ / doi.org/10.1016/j.segan.2019.100226

22. Chernyak, O., Kharlamova, G., \& Stavytskyy, A. (2018). Energy perspective 2030 for Ukraine in the context of the EU integration. In S. Mărginean, C. Ogrean, \& R. Orăștean (Eds.), Emerging Issues in the Global Economy. SPBE (pp. 113-129). Springer. https://doi.org/10.1007/ 978-3-319-71876-7_10

23. EnergoVsesvit. (2021). Electricity production in Ukraine for the first half of 2021. https://vse.energy/ news/pek-news/electro/1716-power-generation-202106 (in Ukrainian)

24. Widodo, D.A., Iksan, N., \& Udayanti, E.D. (2021). Renewable energy power generation forecasting using deep learning method. IOP Conference Series: Earth and Environmental Science, 700(1), 012026. https://doi.org/10. 1088/1755-1315/700/1/012026

25. Amasyali, K., \& El-Gohary, N. (2021). Machine learning for occupant-behavior-sensitive cooling energy consumption prediction in office buildings. Renewable and Sustainable Energy Reviews, 142, 110714. https://doi.org/ 10.1016/j.rser.2021.110714

26. Faquir, D., Chouliaras, N., Vlachou, S., Kalopoulou, O. \& Maglaras, L. (2021). Cybersecurity in smart grids, challenges and solutions. AIMS Electronics and Electrical Engineering, 5(1), 24-37. https:/ / doi.org/10.3934/electren g.2021002

27. Zekić-Sušac, M., Mitrović, S., \& Has, A. (2021). Machine learning based system for managing energy efficiency of public sector as an approach towards smart cities. International Journal of Information Management, 58, 102074. https://doi.org/10.1016/j.ijinfomgt.2020.102074

28. Luo, X.J., Oyedele, L.O., Ajayi, A.O., \& Akinade, O.O. (2020). Comparative study of machine learningbased multi-objective prediction framework for multiple building energy loads. Sustainable Cities and Society, 61, 102283. https:// doi.org/10.1016/j.scs.2020.102283

29. Kong, W., Dong, Z.Y., Jia, Y., Hill, D.J., Xu, Y., \& Zhang, Y. (2017). Short-term residential load forecasting based on LSTM recurrent neural network. IEEE Transactions on Smart Grid, 10(1), 841-851. https://doi.org/10. 1109/TSG.2017.2753802

30. Mathas, C.M., Grammatikakis, K.P., Vassilakis, C., Kolokotronis, N., Bilali, V.G., \& Kavallieros, D. (2020). Threat landscape for smart grid systems. In Proceedings of the 15th International Conference on Availability, Reliability and Security (ARES '20) (pp. 1-7). Association for Computing Machinery. https:/ / doi.org/10.1145/3407023.3409229

31. Zhang, Y., Huang, T., \& Bompard, E.F. (2018). Big data analytics in smart grids: a review. Energy Informatics, 1(1), 8. https:// doi.org/10.1186/s42162-018-0007-5

32. Zbitnieva, M., Okhrimenko, V., \& Nosenko, I. (2021). System aspects of scientific researches in power engineering. Lighting Engineering \& Power Engineering, 60(1), 31-38. https://doi.org/10.33042/2079-424X.2021.60. 1.05 
33. Henri, G., Levent, T., Halev, A., Alami, R., \& Cordier, P. (2020). pymgrid: An open-source python microgrid simulator for applied artificial intelligence research. ArXiv Preprint. https:/ / arxiv.org/abs/2011.08004v1

34. Ehsan, A., \& Yang, Q. (2018). Optimal integration and planning of renewable distributed generation in the power distribution networks: A review of analytical techniques. Applied Energy, 210, 44-59. https://doi.org/10. 1016/j.apenergy.2017.10.106
35. Ziefle, M., Brell, T., Philipsen, R., Offermann-van Heek, J., \& Arning, K. (2019). Privacy issues in smart cities: insights into citizens' perspectives toward safe mobility in urban environments. In G. Dartmann, H. Song, \& A. Schmeink (Eds.), Big Data Analytics for Cyber-Physical Systems (pp. 275-292). Elsevier. https://doi.org/10.1016/ B978-0-12-816637-6.00013-0

\title{
Smart Grid технології як концепція інноваційного розвитку енергетики: вихідні пропозиції для розвитку України
}

\author{
Владислав Плюгін, Віталій Тєтєрєв, Анатолій Лапко
}

\begin{abstract}
Анотація. Формування концепції розумної мережі Smart Grid пов' язано із низкою питань, зокрема й теоретикометодологічного характеру. Одна $з$ основних проблем формування такої теорії полягає в побудові ії основи, відправним початком для розробки якої є визначення поняття Smart Grid, як концепції систем поглядів на майбутнє електроенергетики, принципи функціонування та технологічний базис якої зазнає суттєвих змін в порівнянні $з$ характерними рисами для сучасної енергетики. Стаття спрямована на огляд та розробку напрямів та підходів до означення поняття Smart Grid у поєднанні з механізмами машинного навчання, висвітлення їх різноманітного та спільного характеру для вироблення цілісного інноваційного розвитку енергетики. В даній роботі було проведено дослідження енергетичної галузі в Україні. Розглянуто ії ефективність та інноваційний розвиток. Було виділено проблеми при впроваджені технології Smart Grid, які постають при застосуванні альтернативних джерел та систем моніторингу й адміністрування. Визначаються шляхи математичної постановки задачі оптимізації Smart Grid з використанням підходу Data Science на базі системи машинного навчання і нейронних мереж. А саме, методи обробки великих даних (Big Data), інтелектуального аналізу даних (Data Mining), статистичні методи, методи штучного інтелекту, а також машинне навчання (Machine Learning). Проектування i розробка баз даних та прикладного програмного забезпечення буде відбуватися за допомогою методу Data Science. Smart-технології переймуть процеси контролю, обліку та діагностики активів, що забезпечить перспективні можливості самовідновлення енергосистеми, а також ефективний режим експлуатації основних фондів. При впровадженні технологій Smart Grid для української електроенергетики відбудуться значні принципові зміни. Це перехід від централізованих методів генерації і передачі електроенергії до розподілених мереж 3 можливістю управління об'єктами енерго-виробництва і топологією мережі в будь-якій точці, в тому числі на рівні споживача. Заміна централізованого прогнозування попиту за методологією активного впливу споживача стає елементом і суб'єктом системи управління. Відбудеться побудова високопродуктивної інформаційнообчислювальної інфраструктури, як ядра енергетичної системи. Такий підхід формує передумови для широкого впровадження нових пристроїв, що підвищують маневреність і керованість обладнання. Створення операційних додатків наступного покоління (SCADA/EMS/NMS) дозволяє використовувати інноваційні алгоритми і методи управління енергосистемою, у тому числі їі новими активними елементами.
\end{abstract}

Ключові слова: розумна мережа, інтелектуальне вимірювання, SCADA, наука про дані, великі дані, машинне навчання.

\section{NOTES ON CONTRIBUTORS}

Vladyslav Pliuhin

vladyslav.pliuhin@kname.edu.ua

Vitaliy Teterev

vitaliy.teterev@kname.edu.ua

Anatolii Lapko

lapko.an@ua.energy
D.Sc., Professor

Department of Urban Power Supply Systems and Power Consumption

O. M. Beketov National University of Urban Economy in Kharkiv, Kharkiv, Ukraine

(iD) https://orcid.org/0000-0003-4056-9771

p https://publons.com/researcher/F-4627-2018

https:/ / scopus.com/authid/ detail.uri?authorId=57204286328

Postgraduate student

Department of Urban Power Supply Systems and Power Consumption

O. M. Beketov National University of Urban Economy in Kharkiv, Kharkiv, Ukraine

(iD) https://orcid.org/0000-0002-0841-9055

Head of the Renewable Energy Sources Forecasting Team

JSC National Power Company Ukrenergo, Kyiv, Ukraine

D https://orcid.org/0000-0003-3337-3797 